\title{
A high resolution Mirnov array for the Mega Ampere Spherical Tokamak
}

\author{
M. J. Hole, ${ }^{1,2, a)}$ L. C. Appel, ${ }^{1}$ and R. Martin ${ }^{1}$ \\ ${ }^{1}$ Euratom/CCFE Fusion Association, Culham Centre for Fusion Energy, Abingdon, Oxon OX14 3DB, \\ United Kingdom \\ ${ }^{2}$ Research School of Physics and Engineering, Australian National University, Canberra, Australian Capital \\ Territory 0200, Australia
}

(Received 9 September 2009; accepted 16 November 2009; published online 31 December 2009)

Over the past two decades, the increase in neutral-beam heating and $\alpha$ particle production in magnetically confined fusion plasmas has led to an increase in energetic particle driven mode activity, much of which has an electromagnetic signature which can be detected by the use of external Mirnov coils. Typically, the frequency and spatial wave number band of such oscillations increase with increasing injection energy, offering new challenges for diagnostic design. In particular, as the frequency approaches the megahertz range, care must be taken to model the stray capacitance of the coil, which limits the resonant frequency of the probe; model transmission line effects in the system, which if unchecked can produce system resonances; and minimize coil conductive shielding, so as to minimize skin currents which limit the frequency response of the coil. As well as optimizing the frequency response, the coils should also be positioned to confidently identify oscillations over a wide wave number band. This work, which draws on new techniques in stray capacitance modeling and coil positioning, is a case study of the outboard Mirnov array for high-frequency acquisition in the Mega Ampere Spherical Tokamak, and is intended as a roadmap for the design of high frequency, weak field strength magnetic diagnostics. () 2009 American Institute of Physics. [doi:10.1063/1.3272713]

\section{INTRODUCTION}

In toroidal magnetically confined plasmas, electromagnetic oscillations range in period from the plasma evolution time scale and bulk rotation speed (milliseconds) through to the electron cyclotron period (microseconds). An important and well studied class of modes for fusion power are shear Alfvén eigenmodes (AEs), typically in the frequency range of hundreds of kilohertz. ${ }^{1}$ At large amplitudes, shear Alfvén modes can eject the resonant energetic particles driving the mode from confinement, ${ }^{2}$ short circuiting the collisional heating mechanism, and thereby posing a risk to fusion power. At higher energy and frequency there is also growing evidence for compressional AEs. ${ }^{3}$ In addition to Alfvénic modes, which are modes of the background thermal plasma driven unstable by the energetic populations, there also exist nonperturbative modes [energetic particle modes (EPMs)], which exist only in the presence of an energetic population. ${ }^{4}$ Such modes have frequencies characteristic of energetic particle motion, such as transit, bounce, and precession frequencies. Regardless of mode type, the detection and characterization of electromagnetic mode activity remains important for fusion, as mode evidence can motivate scientific discovery, help characterize the equilibrium through techniques such as magnetohydrodynamics spectroscopy, and in the case of shear Alfvén waves, quantify the level of anomalous transport.

AEs are of particular importance to the compact or

\footnotetext{
${ }^{\text {a) }}$ Author to whom correspondence should be addressed. Electronic mail: matthew.hole@anu.edu.au.
}

spherical torii concept. Such designs feature lower toroidal field and hence lower Alfvén velocity, as well as large gaps in the continuum leading to reduced continuum damping. ${ }^{5}$ This combination leads to increased drive of AEs in the spherical tokamak (ST). In the Mega Ampere Spherical Tokamak (MAST), a wide range of AE and EPM activity has now been observed, including compressional AEs (Ref. 3) and their interaction with tearing modes, ${ }^{6}$ Alfvén cascades and ellipticity induced AEs driven by an active antenna, ${ }^{7}$ EPMs ${ }^{8}$ frequency sweeping hole clumps, ${ }^{9}$ and global AEs driven in Ohmic plasmas. ${ }^{10}$ These modes, and the tearing modes with which they can interact, vary in frequency from $10 \mathrm{kHz}$ up to several megahertz. To date, the observed poloidal $(m)$ and toroidal $(n)$ mode numbers range are in the range $|n|<20,|m|<20$.

Prior to 2003, MAST did not have any dedicated high frequency magnetic diagnostic coils. High frequency magnetic fluctuation measurements were made by nonintegrating a small number of equilibrium Mirnov coils. These coils, which were designed as field integrators, and thus optimized to detect changes in the equilibrium field, suffered a number of severe limitations when used to measure high frequency field fluctuations: the alias spatial toroidal mode number was $n=6$, the inboard coil shielding attenuated the signal by a factor of 0.005 at a frequency of $100 \mathrm{kHz}$, and the coil cabling was not terminated, resulting in system resonances at $300 \mathrm{kHz}$ and its harmonics. With this background in mind, there was a need for a dedicated high frequency Mirnov array of three-axis probes to measure mode strength, mode number, mode polarization, and mode orientation relative to the equilibrium field. The design objectives of this new array, 


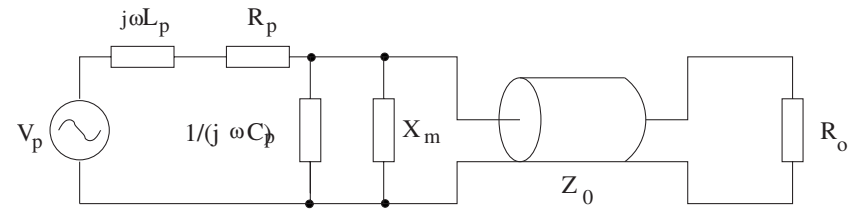

FIG. 1. Equivalent impedance model of the Mirnov coil, matching element $X_{m}$, transmission line of impedance $Z_{0}$, and resistive termination of impedance $R_{0}$ across which the A/D converter samples.

later to be known as the outboard Mirnov array for highfrequency acquisition (OMAHA) for MAST were as follows: (D1) toroidal mode number identification up to a Nyquist number $n_{c}$ of 20; (D2) phase path length across each coil less than $5^{\circ}$ for maximum toroidal mode number; (D3) first selfresonant frequency of Mirnov coil, $f_{r}$ above $5 \mathrm{MHz}$; (D4) detected voltage maximized at $100 \mathrm{kHz}$; and (D5) impedance matched at output.

In this work we design the three-coil probe array to meet design objectives (D1) and (D2) for all three $\delta B$ coils: $\delta B_{R}$, $\delta B_{Z}$, and $\delta B_{\phi}$. Design objectives (D3), (D4), and (D5) are optimized for the $\delta B_{R}$ coil, and the design of the $\delta B_{Z}$ and $\delta B_{\phi}$ coils modified slightly from the $\delta B_{R}$ design to meet engineering constraints. Our work draws on new techniques in stray capacitance calculation ${ }^{11}$ and transmission line modelling, ${ }^{12}$ and presents a new technique for coil placement to maximize the resolving power of an array with a minimum number of coils. We intend it as a roadmap for the design of high frequency, weak field strength magnetic diagnostics. The remainder of this paper is organized as follows: Sec. II introduces the equivalent circuit of the diagnostic system and focuses on optimizing the frequency response. The section spans impedance matching, coil specification, self-resonance, transmission line modeling, optimization, fault-analysis, and coil shielding. Next, Sec. III inverts a recently developed Fourier-singular value decomposition (SVD) mode analysis technique ${ }^{13}$ to optimize the coil positions in MAST, and Sec. IV shows Alfvénic activity measured by the new array in MAST. Finally, Sec. V contains concluding remarks.

\section{OMAHA COIL DESIGN}

Figure 1 is an equivalent circuit of a magnetic fluctuation coil, transmission line, and digitizer system with matching elements. On the left hand side, the magnetic fluctuation in the coil, of strength $B_{c}(\omega)$ with $\omega$ the angular frequency, produces a probe voltage $V_{p}(\omega)=j \omega A N B_{c}(\omega)$, with $A$ the probe cross-sectional area, $N$ the number of turns, and $j$ the complex number such that $j^{2}=-1$. Up to the first selfresonant frequency of the coil $f<f_{r}$, the coil can be adequately modeled as an $R L C$ circuit, with probe selfinductance $L_{p}$, resistance $R_{p}$, and stray capacitance $C_{p}$. A parallel impedance $X_{m}(\omega)$, inserted for matching purposes, connects the Mirnov coil to a lossless transmission line of impedance $Z_{0}$, the output of which is connected to a load $R_{0}$, across which the digitizer samples.

Impedance matching between the transmission line and digitizer can be accomplished by setting $R_{0}=Z_{0}$. In the ideal system, no further matching is required, as only the coil drives current through the transmission line. In reality, further impedance mismatches may arise due to the vacuum feed-through, the cable to digitizer termination, and possibly imperfections in the cable itself. The effect of such mismatches can be minimized by the introduction of a matching circuit between coil and cable, represented by $X_{m}$ in Fig. 1 . Unlike the cable, the impedance of the coil is frequency dependent, and so a match using resistive circuit components can only be achieved at one frequency. Matching over a frequency range could be accomplished by the introduction of reactive circuit elements (additional inductance or capacitance), but the effect of these would also be to modify existing and/or introduce new resonances into the circuit. Finally, active circuit element matching is problematic, owing to the conditions inside the vessel. A resistor is thus proposed as the coil-cable matching element. The matching condition to eliminate reflection of any backward propagating waves (i.e., those reflected from the digitizer toward the coil) is

$$
\left(1 / j \omega C_{p}\right)\left\|\left(R_{p}+j \omega L_{p}\right)\right\| X_{m}=Z_{0},
$$

which at frequencies well below the first self-resonant frequency of the probe reduces to $X_{m} \approx Z_{0}$. Here, the $\|$ notation represents the parallel combination of impedance, for example, $X_{m} \| Z_{0}=\left(1 / X_{m}+1 / Z_{0}\right)^{-1}$. We consider two impedance matching cases: (i) $X_{m}=\infty$ and (ii) $X_{m}=Z_{0}$.

For $\omega<\omega_{r}$, the transfer function $H_{V, \delta B}=V / \delta B$ can be written as

$$
\begin{aligned}
H_{V, \delta B} & =\frac{1 /\left(j \omega C_{p}\right)\left\|X_{m}\right\| Z_{0}}{R_{p}+j \omega L_{p}+1 /\left(j \omega C_{p}\right)\left\|X_{m}\right\| Z_{0}} \times j \omega A N, \\
& \approx \frac{W}{R_{p}+j \omega L_{p}+W} \times j \omega A N, \quad \omega \ll \omega_{r},
\end{aligned}
$$

where $W=X_{m} \| Z_{0}$. Using this circuit model, we optimize the coil and cable specification. The section is organized as follows: Sec. II A chooses the coil dimension to meet the phase path length requirement; Sec. II B selects the coil wire dimension and dielectric coating thickness to meet the inductor frequency design limit (D1); Sec. II C computes the dependence of the transfer function with the number of turns; Sec. II D characterizes the cable; Sec. II E completes the design of the probe; Sec. II F details the construction of each threeprobe assembly; and finally, Sec. II G discusses the effect of the thin graphite shielding of each probe.

\section{A. Coil dimensions}

Estimates of the maximum coil dimensions can be obtained by meeting objectives of phase selectivity $\Delta \Phi<5^{\circ}$ while maximizing the signal-to-noise ratio. That is, the coil length in the toroidal and poloidal directions $\left(l_{\phi}\right.$ and $l_{\theta}$, respectively) is limited to the fraction $\Delta \Phi / 2 \pi$ of the wavelength at the maximum mode number

$$
l_{\phi} \lesssim \frac{R_{\mathrm{wall}} \Delta \phi}{n_{c}},
$$

where $\Delta \Phi$ is the phase variation of the signal across the coil, and $\Delta \phi$ is the toroidal phase path length subtended by the coil. Design requirement (D1) stipulated that the array be capable of resolving mode numbers up to $n_{c}=20$. For two 
coils, the Nyquist spatial aliasing number is $360 /\left(2 n_{c}\right)=9^{\circ}$. A phase selectivity of $5^{\circ}$ is roughly half the Nyquist spacing distance for the maximum mode number, and so the maximum error in phase between two coils is comparable to the Nyquist spacing. We also remark that the phase selectivity constraint on $\Delta \phi$ used to set the maximum dimension of the coil is based on a simple geometric scaling. The actual phase selectivity of the coil is however typically much better than $5^{\circ}$, as the coil measures the flux through the coil, not the oscillating field at the coil center. If, for example, the instantaneous phase of the oscillating field at the center of the coil is $\Phi_{0}$, and the phase difference $\Phi-\Phi_{0}$ of the perturbed field within the coil is an odd function, then the phase error will be zero.

For MAST, $\quad R_{\text {wall }} \simeq 2$ and $R_{\text {mag }} \simeq 0.9, \quad$ yielding $l_{\phi} \lesssim 10.5 \mathrm{~mm}$, In order to minimize variability between the different coils, we elect to use a compactly wound doublelayer coil. Compact winding avoids the need for a grooved former, and a double-layer is chosen so as to eliminate the need for a return wire, which would otherwise be present in the single-layer wound coil. For a double-layer coil the dense packing assumption $l=N d / 2$ can be made, with $d$ the diameter of the coil wire and $l$ the length of the coil. For design purposes, an expression for the coil self-inductance, which includes the effect of fringing fields, is available from Wheeler, ${ }^{14}$

$$
L_{p}=\frac{39.4 r^{2} N^{2}}{9 r+10 l},
$$

with $L_{p}$ in $\mu \mathrm{H}$. Wheeler quotes this expression as valid to within $1 \%$ providing $l>0.8 r$, the number of turns $N \gg 1$, the coil spacing is not too great, and the skin-effect is unimportant. Inspection of Eqs. (5) and (3) shows that $H_{V, \delta B}$ can be maximized by maximizing $A$, and hence $r$, so we choose $r=10 \mathrm{~mm}$.

Expressions for the frequency dependence of the coil resistance are available from McLachlan. ${ }^{15}$ McLachlan solves the current diffusion equation for the axial current density of a circular cross-section wire, integrates to give the total current, and identifies resistive and reactive components of the complex current density. The resistance per unit length can then be expressed as

$$
\frac{R_{p}}{R_{0}}=\frac{k a}{2} \Re\left[\frac{J_{0}\left(k a j^{3 / 2}\right)}{J_{1}\left(k a j^{3 / 2}\right)} e^{j 3 \pi / 4}\right],
$$

where $k=\sqrt{\mu_{0} \sigma \omega}, R_{0}=N l_{t} /\left(\sigma \pi a^{2}\right), \sigma$ is the conductivity of the wire, $\mathfrak{R}$ denotes the real part, and $J_{0}$ and $J_{1}$ are Bessel functions of the first kind of order 0 and 1, respectively.

\section{B. Stray capacitance and wire selection}

To calculate the stray capacitance $C_{p}$, and thereby afford a design prediction of the self-resonant frequency $\omega_{r} \approx 1 / \sqrt{L_{p} C_{p}}$, we draw on the calculation technique developed by Hole and Appel. ${ }^{11}$ In that work, a recursive circuit analysis yielded the low frequency response of the coil, and hence an estimate of the self-resonant frequency. A principal finding was that the stray capacitance of a densely packed coil was in the range $1.45 C_{t t} \leq C_{p} \leq 1.7 C_{t t}$, with $C_{t t}$ the turn-

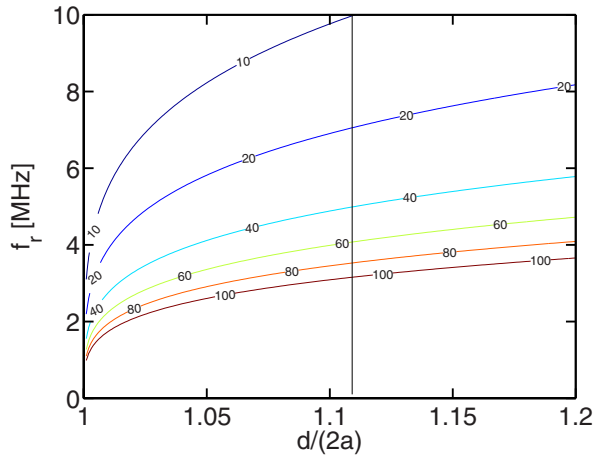

FIG. 2. (Color online) Contour plot of coil self-resonant frequency for different inductor values (in $\mu \mathrm{H}$ ) and as a function of wire plus insulation radius $d$ to wire radius $a$. The dashed line indicates the design selection.

to-turn capacitance of each winding. The lower and upper estimates are for without a shield, and with a shield, respectively. The turn-to-turn capacitance is given by

$$
C_{t t}=\frac{\pi l_{t} \varepsilon_{d}}{\cosh ^{-1}\left(\frac{d}{2 a}\right)},
$$

with $l_{t}=2 \pi r$ the length of wire in one turn, $\varepsilon_{d}$ the permittivity of the dielectric coating of the wire, $d$ the width of the wire plus dielectric coating, and $a$ the wire radius. ${ }^{16}$

Figure 2 is a plot of the self-resonant frequency $f_{r}=\omega_{r} /(2 \pi)$ against the ratio of wire plus dielectric coating thickness $d$ to wire diameter $2 a$, for different inductor values $L_{p}$, and for a polyamide/imide insulator of relative permittivity $\varepsilon_{d} / \varepsilon_{0}=4.7$. The plot shows $f_{r}$ increases with increasing $d / a$. Using the dense packing assumption $d=2 l / N$, a simple analysis also shows that for given $l,\left|H_{V, \delta B}\right|$ decreases with increasing $d$, suggesting $d$ be minimized. To simultaneously maximize $f_{r}$ and $\left|H_{V, \delta B}\right|$, then $a$ should also be minimized. Coupling these trends with the engineering constraints $2 a \geq 0.55 \mathrm{~mm}$ and $t \geq 0.030 \mathrm{~mm}$ together with the requirement $f_{r}>5 \mathrm{MHz}$ suggests the design $2 a=0.55 \mathrm{~mm}$ and $t=0.030 \mathrm{~mm}$.

\section{Number of turns}

The number of turns can be optimized by extremizing $\left|H_{V, \delta B}\right|$ with respect to $N$. As in other works, ${ }^{17}$ simple scaling laws can be recovered when $R_{p} \ll W$ and $L_{p} \propto N^{2}$ is assumed. Thus, using Eq. (3) for $H_{V, \delta B}$, the equation $\partial\left|H_{V, \delta B}\right| / \partial N=0$ can be solved for $L_{p}$, yielding the condition $L_{p}=W / \omega$. Solving $L_{p}=W / \omega$ for $N$ and substituting into $H_{V, \delta B}$ gives

$$
H_{V, \delta B}=\frac{j A}{1+j} \sqrt{\frac{\omega W(9 r+10 l)}{39.4 r^{2}}},
$$

and so $\left|H_{V, \delta B}\right|$ is largest when $W$ is maximized. Further design requires knowledge of the electrical characteristics of the transmission line.

\section{Cable}

For the new array, an experimental triaxial cable was trialed, with the outermost shield grounded. The triaxial cable offers the advantages of complete electrostatic shielding, as well as high frequency electromagnetic shielding. Im- 


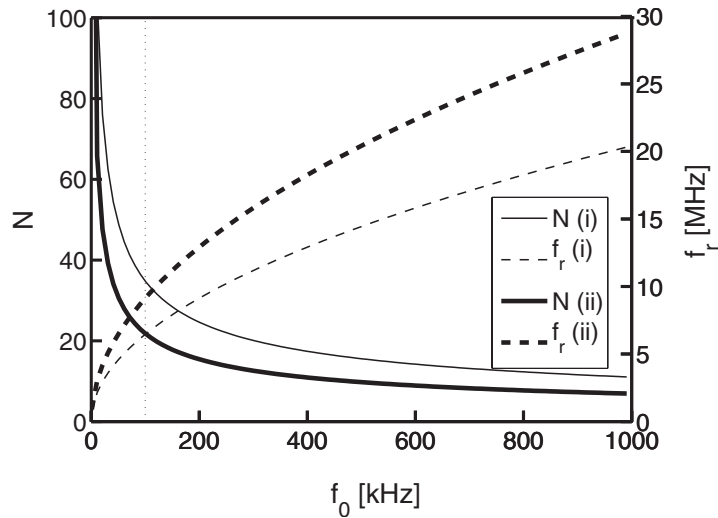

FIG. 3. Optimized number of coil turns (left axis) and self-resonant frequency (right axis) as a function of design frequency $f_{0}$, and for the two cases of Table I. The dashed line indicates the design optimization frequency of $100 \mathrm{kHz}$.

pedance measurements of the triaxial cable using a RSZVx Rhodes and Schwarz ${ }^{18}$ network analyzer yield $Z_{0}=13.5 \Omega$.

\section{E. Design choices}

With the characteristics of the transmission line determined, the Mirnov coil design can now be completed. Using $L_{p}=W / \omega$, we solve Eq. (5) for $N$, given $r$ and $A$, and hence compute $H_{V, \delta B}\left(\omega_{0}\right)$ using Eq. (2) for the different terminations, with $\omega_{0}$ the operating angular frequency. The coil length is then determined by $l=N d / 2$.

Figure 3(a) shows the number of turns $N$ (left axis) and self-resonant frequency $f_{r}$ (right axis), as a function of design frequency and for different terminations. The inverse quadratic scaling of the number of turns $N$ with design frequency $f_{0}=\omega_{0} /(2 \pi)$ and quadratic scaling with impedance $W$ (i.e., $N \propto \sqrt{W / \omega}$ ) is a consequence of the optimization condition $L_{p}=W / \omega$, together with the expression for $L_{p}$, Eq. (5). Table I lists the optimized circuit parameters for the different terminations. Our final design selection is motivated by the need to minimize reflections in the system over the frequency range of interest: this suggests design (ii).

It is worth noting that there exists a $10 \%$ discrepancy between $L_{p}=W / \omega$ and the tabulated design choices in Table I. The difference arises because the scoping dependence $L_{p} \propto N^{2}$, which was used in Sec. II C to find the optimization condition $L_{p}=W / \omega$, does not capture the full inductor dependence with the number of turns $N$ through the dense packing assumption $l=N d / 2$.

TABLE I. Optimized design at $f_{0}=100 \mathrm{kHz}$ for the different types of termination.

\begin{tabular}{lcc}
\hline \hline Parameter & Case (i) & Case (ii) \\
\hline$W$ & $13.5 \Omega$ & $6.7 \Omega$ \\
$N$ & 32 & 22 \\
$l$ & $9.8 \mathrm{~mm}$ & $6.7 \mathrm{~mm}$ \\
$r$ & $10 \mathrm{~mm}$ & $10 \mathrm{~mm}$ \\
$L_{p}$ & $22 \mu \mathrm{H}$ & $12 \mu \mathrm{H}$ \\
$C_{p}$ & $25 \mathrm{pF}$ & $25 \mathrm{pF}$ \\
$f_{r} \approx 1 / \sqrt{L_{p} C_{p}}$ & $6.8 \mathrm{MHz}$ & $9.6 \mathrm{MHz}$ \\
$\left|H_{V, \delta B}\left(\omega_{0}\right)\right|$ & 4400 & 2900 \\
\hline \hline
\end{tabular}

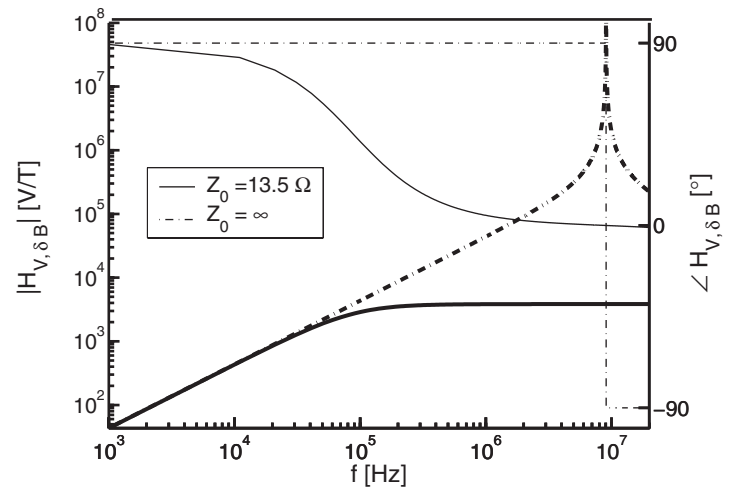

FIG. 4. Magnitude (left axis) and phase (right axis) of the transfer function $H_{V, \delta B}$ for the coil design selection (ii), and in the absence of a shield. Heavy and light lines denote magnitude and phase, respectively. Also shown is the transfer function when the cable impedance is infinite (dashed line).

Figure 4 shows the transfer function for design (ii) across the entire frequency range. When the transmission line is present, the transfer function saturates in magnitude, and the phase of $H_{V, \delta B}$ approaches zero. If no transmission line cabling is present, such that $W \rightarrow \infty$, then the voltage transfer function does not roll off at $100 \mathrm{kHz}$, but instead ramps up to the first self-resonant frequency of the coil, at which the phase changes by $\pi$. The open circuit termination corresponds to a standard $R L C$ circuit model, which was used in Sec. II B to determine the first self-resonant frequency of the coil, and hence select the wire diameter.

\section{F. Construction}

Figure 5 is the final equivalent circuit for each probe, showing shield and ground connections, and the circuit connection for the triaxial cable. The tails of each coil were connected to ground by a $6.7 \Omega$ resistor. A triaxial cable then connects each coil to the digitizer input, which is terminated in parallel by a $13.5 \Omega$ resistor.

Figure 6(a) is a photo of the three-axis $\delta B$ probes, which are wound on a common ceramic former. For each former, the windings of different orientation coils overlap, leading to a slightly different $N A$ for each coil: for $\delta B_{\phi}, N A=6.5$ $\times 10^{-3} \mathrm{~m}^{-2}$, for $\delta B_{Z}, N A=7.5 \times 10^{-3} \mathrm{~m}^{-2}$, and for $\delta B_{R}, N A$ $=8.8 \times 10^{-3} \mathrm{~m}^{-2}$. To eliminate difference in propagation delays between different coils, the cable lengths for all of the coils was made identical.

The coils were mounted at the end of a $30-\mathrm{cm}-$ long, $17.5 \mathrm{~mm}$ radius cylindrical $\mathrm{Al}_{2} \mathrm{O}_{3}$ ceramic test tube, which was bolted to the vessel wall [see Fig. 6(b)]. This suspension minimized the effect of induced vessel wall currents. To minimize injection of high $Z$ impurities from the test tube,

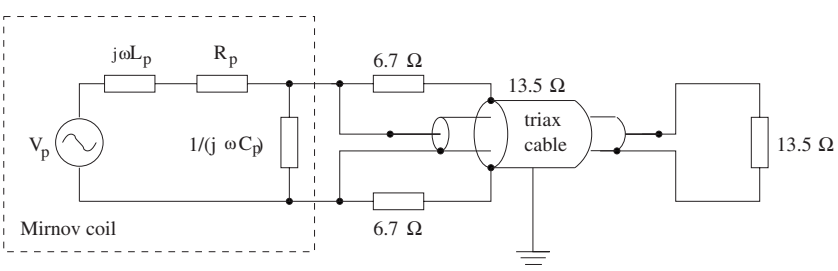

FIG. 5. An equivalent circuit of the final probe design for each coil, showing ground connections and the circuit connection for the triaxial cable. 


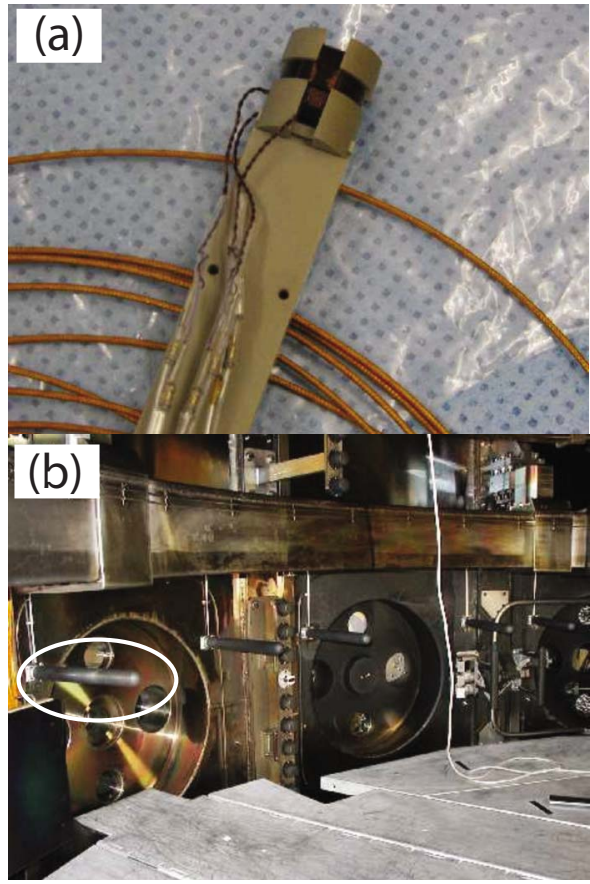

FIG. 6. (Color online) Images of (a) the three-axis probe head and (b) the probe test tube bolted to the vessel wall. The leftmost OMAHA probe is identified by the white ellipse. Five OMAHA probes are visible.

each test tube was coated with a $0.2 \mathrm{~mm}$ layer of colloidal graphite. The effect of this graphite layer on the transfer function is calculated in Sec. II G.

\section{G. Shield}

Compared to the $\delta B_{Z}$ and $\delta B_{\phi}$ coils, the shared flux between the $\delta B_{R}$ coil and the shield will be largest, as the $\delta B_{R}$ coil axis and shield axis are parallel and the shield encases the coil. The $\delta B_{R}$ coil will also feature the lowest shield resistance.

Following Strait, ${ }^{19}$ we model the effects of the graphite shield as the secondary winding of a one-turn solenoidal transformer. The secondary winding has self-inductance $L_{S}$ and resistance $R_{S}$, and the mutual inductance between the coil and shield is $M$. Our analysis departs from Strait, who neglects capacitive effects, by inclusion of the parallel capacitance of the coil-shield system, $C_{p}$. With this correction, the impedance across the terminals of the probe is then given by

$$
\begin{aligned}
Z_{p}= & \left\{R_{p}\left[1+K^{2} \frac{\left(\omega \tau_{s}\right)\left(\omega \tau_{p}\right)}{1+\left(\omega \tau_{s}\right)^{2}}\right]\right. \\
& \left.+j \omega L_{p}\left[1-K^{2} \frac{\left(\omega \tau_{s}\right)^{2}}{1+\left(\omega \tau_{s}\right)^{2}}\right]\right\} \| 1 /\left(j \omega C_{p}\right),
\end{aligned}
$$

where $\tau_{p}=L_{p} / R_{p}$ is the probe time constant, $\tau_{s}=L_{s} / R_{S}$ is the shield time constant, and $K^{2}=M^{2} /\left(L_{p} L_{s}\right) \approx A_{p} / A_{s}$ is the coupling coefficient between probe and shell, with $A_{P}$ and $A_{S}$ are the cross-section of the probe and shell. Strait ${ }^{19}$ composed the voltage transfer function $H(\omega)=V_{p} / V_{\text {in }}$ in the form

$$
H(\omega)=H_{s}(\omega) H_{p}(\omega)
$$

with $H_{s}(\omega)=\left(1+j \omega \tau_{s}\right)^{-1}$ and $H_{p}(\omega)=Z_{0} /\left(Z_{p}+Z_{0}\right)$. Here, the term $H_{s}(\omega)=\left(1+j \omega \tau_{s}\right)^{-1}$ describes the attenuation of the magnetic field by the shell, and includes eddy currents in the shield induced by the field. In the analysis of Strait, ${ }^{19}$ the second term $H_{p}(\omega)=Z_{0} /\left(Z_{p}+Z_{0}\right)$ describes the voltage divider created by the input impedance of the external circuit $Z_{0}$ (or matched transmission line), and includes eddy currents in the shell induced by currents in the coil. In the limit that $Z_{p} \simeq R_{p}+j \omega L_{p}$ the effect of the shield is to attenuate the transfer function by the fraction $H_{s}(\omega)$. The voltage transfer function $H$ is related to the signal transfer function $H_{V, \delta B}$ by $H_{V, \delta B}=H j \omega A$.

Each OMAHA three-probe shield has radius $r_{s}$ $=17.5 \mathrm{~mm}$, length $l_{s}=0.3 \mathrm{~m}$, and is coated with $t_{s}=0.2 \mathrm{~mm}$ of graphite. An expression for the ac shield resistance for a tube cylinder of inner radius $b$ and outer radius $a$ is available from McLachlan, ${ }^{15}$

$$
\begin{aligned}
\frac{R_{s}}{R_{0}}= & \frac{k}{2}\left(\frac{a^{2}-b^{2}}{a}\right) \\
& \times \Re\left[\frac{i^{3 / 2} J_{0}\left(k a i^{3 / 2}\right) K_{1}\left(k b i^{1 / 2}\right)-i^{5 / 2} J_{1}\left(k b i^{3 / 2}\right) K_{0}\left(k a i^{1 / 2}\right)}{J_{0}\left(k a i^{3 / 2}\right) K_{1}\left(k b i^{1 / 2}\right)-J_{1}\left(k b i^{3 / 2}\right) K_{0}\left(k a i^{1 / 2}\right)}\right],
\end{aligned}
$$

where $K_{0}$ and $K_{1}$ are modified Bessel functions of order 0 and 1 , and the dc resistance of the shield is $R_{0}=l_{s} /\left[\sigma_{c} \pi\left(a^{2}\right.\right.$ $\left.-b^{2}\right)$ ], where $\sigma_{c}$ is the conductivity of the graphite. As with Eq. (6), the resistance increases with increasing frequency, and the current becomes more localized to the surface of the shield. The radial field varies over the radial length of the shield, particularly near the vessel wall where the field is terminated. As an upper limit to the shield inductance we assume a homogeneous field over half the length of the shield. Using Eq. (5) with $l=l_{s} / 2$ yields $L_{s}=7.3 \mathrm{nH}$ and $K \approx \sqrt{A_{p} / A_{s}}=0.54$. Using Eq. (11) with $b=17.5 \mathrm{~mm}$, $a=17.7 \mathrm{~mm}$, and $\sigma_{C}=8.0 \times 10^{-6} \Omega \mathrm{m}^{-1}$, we compute $R_{0}=0.054 \Omega$ and $R_{S} \approx R_{0}$ for $f<10 \mathrm{MHz}$. Consequently, the shield time constant is $\tau_{s}=L_{s} / R_{s} \approx 1.3 \mu \mathrm{s}$, which is significantly smaller than the probe time constant $0.018<\tau_{p}$ $=L_{p} / R_{p}<0.12 \mathrm{~ms}$.

At the design frequency of $100 \mathrm{kHz}, \omega \tau_{s}=0.084$, and so the attenuation of the magnetic field (as well as the effect of eddy currents in the shield due to the fluctuating field) by the shell is insignificant: the change in $\left|H_{s}\right|$ is 0.003 and the change in phase $\angle H_{s} \approx-4^{\circ}$. The correction terms to $R_{p}$ and $L_{p}$ through Eq. (9) are 2.0 and -0.002 , respectively, and so the correction to $Z_{p}$ is also small. In contrast, at the first self-resonant frequency of the coil, $9.6 \mathrm{MHz}$, the effect of the shield is enormous, with $\omega \tau_{s}=8.4$, the change in $\left|H_{s}\right|$ is 0.88 , and the phase $\angle H_{s} \approx-83^{\circ}$.

Figure 7 shows the magnitude and phase response of the toroidal field coil using the radial shield. We note that the roll off in $\left|H_{s}\right|$ at $f=250 \mathrm{kHz}$ also causes $|H|$ to roll off, compared to the unshielded case. The phase continues to ramp down beyond the peak in $|H|$, and plateaus at $-90^{\circ}$. 


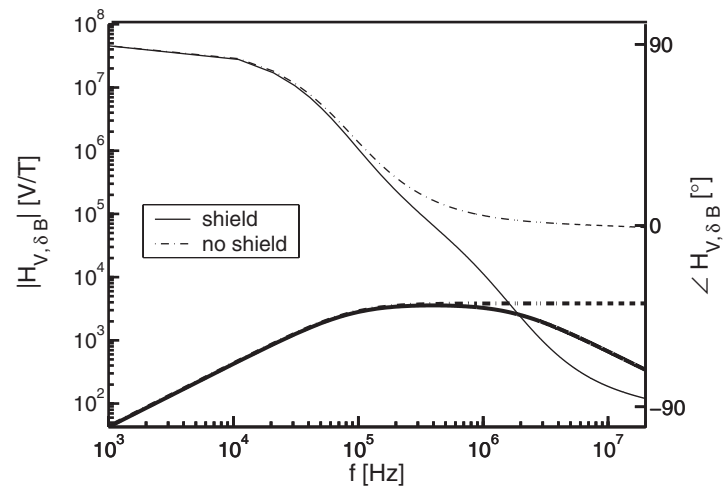

FIG. 7. Magnitude (left axis) and phase (right axis) of the transfer function $H_{V, \delta B}$ for the coil design selection (ii), in the presence of a thin graphite conducting shield (solid line). Heavy and light lines denote magnitude and phase, respectively. Also shown is the transfer function for no shielding (dashed line).

\section{ARRAY POSITIONING}

To date, various numerical search routines have been used to optimize the position of the coils. These include numerical optimization of van Milligen and Jimenez, ${ }^{20}$ who applied functional parametrization to an equilibrium database, yielding expressions for bulk plasma properties as a function of the magnetic field measurements at the coil locations.

Here, we position the toroidal location of the OMAHA probe coils by inverting the Fourier-SVD mode analysis technique of Hole and Appel. ${ }^{13}$ This procedure involves decomposing the calibrated time trace signals $x_{k}$ from the $k$ th magnetic coil at toroidal angle $\phi_{k}$ as a Fourier time and Fourier spatial series, and finding a best fit to this basis. That is,

$$
\begin{aligned}
& x_{k}=\frac{1}{2 \pi} \int e^{-j \omega t} F_{k} d \omega, \\
& =\frac{1}{2 \pi} \int e^{-j \omega t} \sum_{i=1}^{M} \alpha_{i}(\omega) e^{j n_{i} \phi_{k}} d \omega,
\end{aligned}
$$

where $F_{k}$ is the temporal Fourier transform of $x_{k}$ at angular frequency $\omega, \alpha_{i}(\omega)$ is the complex amplitude of each toroidal eigenmode $n_{i}, M$ is the number of toroidal modes of the plasma, and $j^{2}=-1$ is the complex variable. For a coil located at angle $\phi_{k}$, the integrands of Eqs. (12) and (13) equate as follows:

$$
F_{k}=\sum_{i=1}^{M} \alpha_{i} e^{j n_{i} \phi_{k}}
$$

At a particular frequency each measurement provides two constraints via the complex transform Fourier $F_{k}$. The measurement is matched to a set of modes each one of which has three unknowns: magnitude, phase, and eigenmode number. Providing that the signal from different coils is calibrated to a common reference, Eq. (14) can be rewritten as

$$
\mathbf{F}=\boldsymbol{\gamma} \cdot \boldsymbol{\alpha},
$$

for $N$ coils, with

$$
\mathbf{F}=\left(\begin{array}{l}
F_{1} \\
F_{2} \\
\vdots \\
F_{N}
\end{array}\right), \quad \boldsymbol{\gamma}=\left(\begin{array}{lll}
e^{j n_{1} \phi_{1}} & \cdots & e^{j n_{M} \phi_{1}} \\
e^{j n_{1} \phi 2} & \cdots & e^{j n_{M} \phi_{2}} \\
\vdots & & \vdots \\
e^{j n_{1} \phi_{N}} & \cdots & e^{j n_{M} \phi_{N}}
\end{array}\right), \quad \boldsymbol{\alpha}=\left(\begin{array}{l}
\alpha_{1} \\
\vdots \\
\alpha_{M}
\end{array}\right),
$$

where $M$ is the number of distinct eigenmodes in the plasma at angular frequency $\omega$.

In Fourier-SVD mode analysis, Eq. (15) is solved for every unique eigenvalue combination $\Gamma=\left\{n_{1}, n_{2}, \cdots, n_{M}\right\}$ by obtaining the inverse of $\boldsymbol{\gamma}$ through SVD inversion. That is, column-orthonormal $N \times M$ and $M \times M$ matrices $\mathbf{U}$ and $\mathbf{V}$, respectively, and $M$ weights $w_{i}$ are found such that

$$
\boldsymbol{\gamma}=\mathbf{U} \cdot \operatorname{diag}\left(w_{i}\right) \cdot \mathbf{V}^{H},
$$

with $\mathbf{U} \cdot \mathbf{U}^{H}=\mathbf{V} \cdot \mathbf{V}^{H}=\mathbf{I}$, so as to minimize the residual

$$
r=|\boldsymbol{\gamma} \cdot \boldsymbol{\alpha}-\mathbf{F}| /|\mathbf{F}|,
$$

of the solution. Here, the superscript $H$ stands for Hermitian transpose, and denotes the complex conjugate of the transposed matrix. The set of mode complex amplitudes $\boldsymbol{\alpha}$ is then given by

$$
\boldsymbol{\alpha}=\mathbf{V} \cdot \operatorname{diag}\left(1 / w_{i}\right) \cdot \mathbf{U}^{H} \cdot \mathbf{F} .
$$

To find the overall best fit solution, the combination $\Gamma$ is cycled through all possible unique solutions: for $M=1$, the number of unique solutions is $n_{s}=2 n_{c}+1$. In the absence of noise the method yields a set of one or more linearly independent solutions with zero residual, and other nonsolutions with large residuals.

If $M=1$, the matrix of singular values $\operatorname{diag}\left(w_{i}\right)$ and the orthonormal matrix $\mathbf{V}$ become singular, and can thus be written $\operatorname{diag}\left(w_{i}\right)=w e^{j \phi_{w}}$ and $\mathbf{V}=e^{j \phi_{V}}$. The covariance matrix can be evaluated giving $\mu=N$, and thus $w=\sqrt{N}$ and $\phi_{w}=0$. Equation (16) can be solved for $\mathbf{U}$, yielding

$$
\mathbf{U}=\boldsymbol{\gamma} \frac{e^{j \phi_{V}}}{w} \text {. }
$$

Thus, $\boldsymbol{\alpha}$ becomes

$$
\boldsymbol{\alpha}=\frac{e^{j \phi_{V}}}{w}\left(\mathbf{U}^{H} \cdot \mathbf{F}\right)=\frac{1}{w^{2}}\left(\gamma^{H} \cdot \mathbf{F}\right) .
$$

An idealized plasma signal with mode amplitude $f$ and mode number $n$ can be written as

$$
\mathbf{F}=f\left(\begin{array}{l}
e^{j\left(n \phi_{1}+\xi\right)} \\
e^{j\left(n \phi_{2}+\xi\right)} \\
\vdots \\
e^{j\left(n \phi_{N}+\xi\right)}
\end{array}\right),
$$

where $\xi$ is an arbitrary phase reference. For a single mode present, the matrix $\gamma$ can be written as

$$
\gamma=\left(\begin{array}{l}
e^{j(n+\Delta n) \phi_{1}} \\
e^{j(n+\Delta n) \phi_{2}} \\
\vdots \\
e^{j(n+\Delta n) \phi_{N}}
\end{array}\right)
$$

where $-n_{c} \leq n+\Delta n \leq n_{c}$. With these substitutions, Eq. (20) can be rewritten as 


$$
\boldsymbol{\alpha}=\frac{f}{w^{2}} \sum_{i=1}^{N} e^{j\left(-\Delta n \phi_{i}+\xi\right)},
$$

and the normalized residue $r_{s}=r /|\mathbf{F}|$ calculated

$$
\begin{aligned}
\frac{r}{|\mathbf{F}|} & =\left[\frac{(\mathbf{F}-\boldsymbol{\gamma} \cdot \boldsymbol{\alpha})^{H} \cdot(\mathbf{F}-\boldsymbol{\gamma} \cdot \boldsymbol{\alpha})}{\mathbf{F}^{H} \cdot \mathbf{F}}\right]^{1 / 2}, \\
& =\left(1-\frac{1}{N^{2}} \sum_{i=1}^{N} e^{-j \Delta n \phi_{i}} \sum_{i=1}^{N} e^{j \Delta n \phi_{i}}\right)^{1 / 2} .
\end{aligned}
$$

For $\Delta n=0$ the correct mode is identified, and Eq. (23) yields $\boldsymbol{\alpha}=f e^{j \phi}$, whilst Eq. (25) yields $r /|\mathbf{F}|=0$.

We seek a coil placement strategy that maximizes the residue for every other mode (i.e., $\Delta n \neq 0$ ) in the presence of noise. In the presence of weak noise, $\mathbf{F}$ can be rewritten as

$$
\mathbf{F} \equiv f\left(\hat{\mathbf{F}}_{s}+\varepsilon \hat{\mathbf{F}}_{n}\right)=f\left(\begin{array}{l}
e^{j\left(n \theta_{1}+\phi\right)} \\
e^{j\left(n \theta_{2}+\phi\right)} \\
\vdots \\
e^{j\left(n \theta_{N}+\phi\right)}
\end{array}\right)+\varepsilon f\left(\begin{array}{l}
\beta_{1} e^{j \psi_{1}} \\
\beta_{2} e^{j \psi_{2}} \\
\vdots \\
\beta_{N} e^{j \psi_{N}}
\end{array}\right),
$$

where $\varepsilon$ is a measure of the noise-to-signal ratio, the coefficients $\beta$ are the normalized noise amplitudes (normalized such that $\max \left\{\beta_{1}, \beta_{2}, \ldots, \beta_{N}\right\}=1$ ), and the coefficients $\psi$ represent noise angles. The extracted mode amplitudes $\boldsymbol{\alpha}$ can then be written as

$$
\boldsymbol{\alpha} \equiv f\left(\hat{\boldsymbol{\alpha}}_{s}+\varepsilon \hat{\boldsymbol{\alpha}}_{n}\right)=f\left(\frac{1}{w^{2}} \sum_{i=1}^{N} e^{j\left(-\Delta n \theta_{i}+\phi\right)}+\frac{\varepsilon}{w^{2}} \boldsymbol{\gamma} \cdot \hat{\mathbf{F}}_{n}\right)
$$

Solving Eq. (24) for $r^{2}$, and substituting Eqs. (26) and (27) yields a quadratic in $\varepsilon$,

$$
r^{2}=r_{s}^{2}+\varepsilon r_{n 1}+\varepsilon^{2} r_{n 2},
$$

with

$$
\begin{aligned}
& \hat{r}_{n 1}=\frac{\left(\mathbf{F}_{n}-\boldsymbol{\gamma} \cdot \boldsymbol{\alpha}_{n}\right)^{H} \cdot\left(\mathbf{F}_{s}-\boldsymbol{\gamma} \cdot \boldsymbol{\alpha}_{s}\right)+\left(\mathbf{F}_{s}-\boldsymbol{\gamma} \cdot \boldsymbol{\alpha}_{s}\right)^{H} \cdot\left(\mathbf{F}_{n}-\boldsymbol{\gamma} \cdot \boldsymbol{\alpha}_{n}\right)}{\mathbf{F}^{H} \cdot \mathbf{F}}, \\
& \hat{r}_{n 1}=\frac{\mathbf{F}_{n}^{H} \cdot \mathbf{F}_{n}}{\mathbf{F}^{H} \cdot \mathbf{F}},
\end{aligned}
$$

where $\boldsymbol{\alpha}_{s}=f \hat{\boldsymbol{\alpha}}_{s}, \boldsymbol{\alpha}_{n}=f \hat{\boldsymbol{\alpha}}_{n}, \mathbf{F}_{s}=f \hat{\mathbf{F}}_{s}, \mathbf{F}_{n}=f \hat{\mathbf{F}}_{n}$, and where $r_{s}$, the signal residue, is given by Eq. (25).

In general, the dependence of the residue with noise implies that any optimization of coil locations (i.e., maximizing the residue for every other mode, $\Delta n \neq 0$ ) will be sensitive to the detailed properties of the noise [through the $\beta$ and $\psi$ coefficients in Eq. (26)]. That is, if the coil arrangement is defined by $\boldsymbol{\phi}=\left\{\phi_{1}, \phi_{2}, \cdots, \phi_{N}\right\}$, then the optimal coil arrangement will be a function of the signal-to-noise ratio, i.e., $\phi(\varepsilon)$. If, however, the coil arrangement $\boldsymbol{\phi}$ is chosen to maximize the residue for every other mode $(\Delta n \neq 0)$ of an ideal signal $(\varepsilon=0)$, then in the presence of weak noise $(0<\varepsilon$ $\ll 1$ ) the residue for every incorrect solution will also be large (but not necessarily maximized). We assume $\varepsilon r_{n 1}$ $+\varepsilon^{2} r_{n 2}>0$ for all $\delta_{n}>0$, and seek $\lim _{\varepsilon \rightarrow 0} \boldsymbol{\phi}(\varepsilon)=\boldsymbol{\phi}(0)$, an "optimal" solution.

Numerically, we proceed by searching for the arrangement $\boldsymbol{\phi}$ such that $r_{\text {min }}$ is maximized, where

$$
r_{\min }=\min \left[r(\Delta n=1, \boldsymbol{\phi}), r(\Delta n=2, \boldsymbol{\phi}), \cdots, r\left(\Delta n=n_{c}, \boldsymbol{\phi}\right)\right] .
$$

Specifically, we seek solutions for $\boldsymbol{\phi}$ of

$$
\frac{\partial r_{\min }}{\partial \boldsymbol{\phi}}=0,
$$

where $r_{\min }$ is a maximum. Equation (30) represents $N$ equations in $N$ unknowns. The differential operator $\partial / \partial \boldsymbol{\phi}$ can only be taken inside the set with the simultaneous use of the right hand side (RHS) of Eq. (29) to select the minimum element in the set, $r_{\min }$. Even in this case complications arise: (a) solutions to $\boldsymbol{\phi}$ are not single valued: reflections and rotations of $\boldsymbol{\phi}$ will leave $r_{\min }$ unchanged, and so solutions to $\boldsymbol{\phi}$ will be multivalued; and (b) the derivative $\partial r_{\min } / \partial \boldsymbol{\phi}$ may be discontinuous, resulting from a change in element in $r_{\text {min }}$.

We have used Monte Carlo simulation to locate the combination to give maximum $r_{\text {min }}$. This involves seeding the coil arrangement $\boldsymbol{\phi}$, using Eq. (25) to compute the elements $r\left(\Delta n=n_{c}, \boldsymbol{\phi}\right)$, and identifying $r_{\text {min }}$ from Eq. (29). In the limit that the number of coil arrangements, $N_{F}$, approaches infinity (practically, $N_{F}>10^{5}$ ) the coil arrangement with the largest $r_{\text {min }}$ is revealed. An early design requirement was to achieve single mode identification up to $n=n_{c}$ with only two detectors. Numerically, this criterion was satisfied by setting $\phi_{1}$ $=0$ and the second coil positioned at a random angle in the interval $0<\phi_{2}<\pi / n_{c}$, with $n_{c}$ the Nyquist number. The remaining $N-2$ coils were placed at random angles $\phi_{i}$ over the interval $\phi_{i-1}<\phi_{i}<2 \pi$.

On physical grounds, the difference in residue $\Delta r$ must be degenerate to any change in the reference angle $\boldsymbol{\phi} \rightarrow \boldsymbol{\phi}$ $-\left\{\phi_{r}, \ldots, \phi_{r}\right\}$, or any reflection of the coil combination $\boldsymbol{\phi} \rightarrow-\boldsymbol{\phi}$. The degeneracy can be eliminated by defining a subjective mapping, which maps all rotated and reflected versions of $\boldsymbol{\phi}$ to the same vector. Such a transformation is $\boldsymbol{\phi} \rightarrow \widetilde{\boldsymbol{\phi}}$, defined such that the vector formed by the differences $|\Delta \boldsymbol{\phi}|=\left\{\left|\widetilde{\phi}_{2}-\widetilde{\phi}_{1}\right|,\left|\widetilde{\phi}_{3}-\widetilde{\phi}_{2}\right|, \ldots,\left|\widetilde{\phi}_{1}-\widetilde{\phi}_{N}\right|\right\}$, the property 


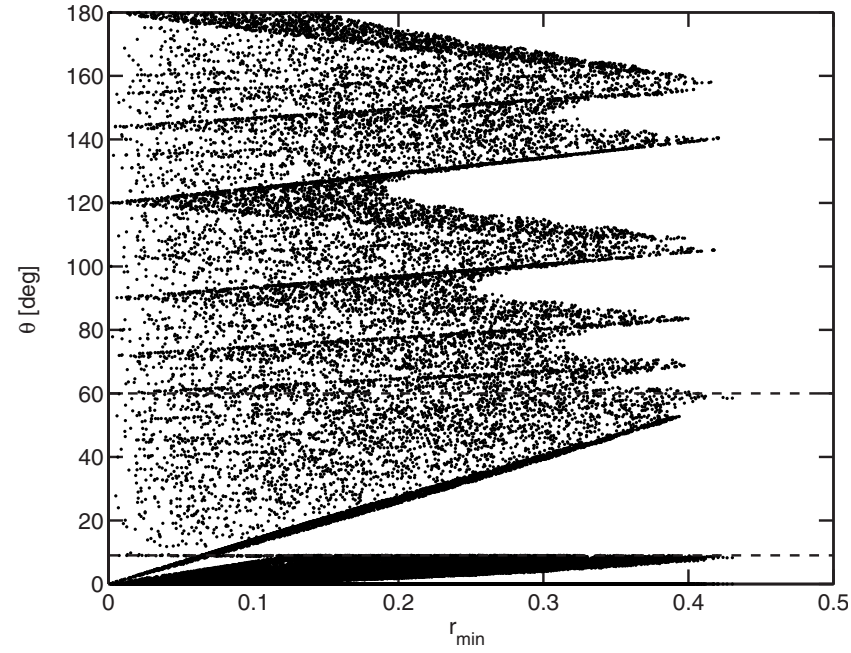

FIG. 8. Plot of coil toroidal angles as a function of $r_{\min }$. Two coils have been placed less than $2 \pi / n_{c}$ apart, to ensure the signal is not aliased. The remaining coils are randomly distributed. Finally, set of angles have been mapped to remove reflection and rotation degeneracy.

$$
\Delta \widetilde{\phi}_{i} \leq \Delta \widetilde{\phi}_{j}
$$

is true up to the first two distinct elements in the differences vector $|\Delta \phi|$.

Figure 8 plots the mapped angles $\boldsymbol{\phi}$ versus $r_{\text {min }}$, the first nonzero residual minimum for $N=3$ and $n_{c}=20$. The reference coil is situated at $\phi=0$, whilst the darker and lighter shaded points correspond to the position of the second and third coils, respectively. The dense set of darker points below $\phi_{c}=\pi / n_{c}$ corresponds to coil spacings, whose separation has been limited to avoid aliasing (only two coils can be placed more than $\phi_{c}$ apart).

The worst coil arrangements, at $r_{s}(\Delta n)=0$, occur on the left of the figure, where two coils are placed at zero separation. The third coil is located at simple rational fractions of $2 \pi$, e.g., $2 m \pi / n$. These solutions are aliased, and so $r_{s}=0$. As the residue $r_{s}(\Delta n)$ increases, the second coil approaches the Nyquist angle, whilst the third coil shifts to lie in the range: $\pi / 6<\phi<\pi / 2$. Qualitatively, this is consistent with a small coil spacing to provide high $n$ identification, and a large coil spacing to provide fine $n$ resolution. Dashed lines show the optimized coil locations, where $r_{\min }$ is largest. For the OMAHA coils, coil placements of $\boldsymbol{\phi}=\left\{0^{\circ}, 9^{\circ}, 60^{\circ}\right\}$ were chosen. In MAST, this corresponded to angles $\boldsymbol{\phi}$ $=\left\{6^{\circ}, 306^{\circ}, 357^{\circ}\right\}$, corresponding to angles $\boldsymbol{\phi}=0^{\circ},-60^{\circ}$, $-9^{\circ}$.

The full set of ten coil positions for OMAHA is

$$
\begin{aligned}
\boldsymbol{\phi}= & \left\{243^{\circ}, 247.5^{\circ}, 267.5^{\circ}, 277.7^{\circ}, 292.5^{\circ}, 306^{\circ},\right. \\
& \left.324^{\circ}, 336^{\circ}, 357^{\circ}, 6^{\circ}\right\} .
\end{aligned}
$$

This coil arrangement was found by repeating the Monte Carlo operations above for $N=10$, and recomputing $r_{\text {min }}$. We have checked that the optimum coil positions for an array of $N$ coils are a subset of the optimum position for an array of $N+1$ coils. That is, the optimal coil arrangement for $N+1$ coils differs from the optimal coil arrangement for $N$ coils by the additional coil.

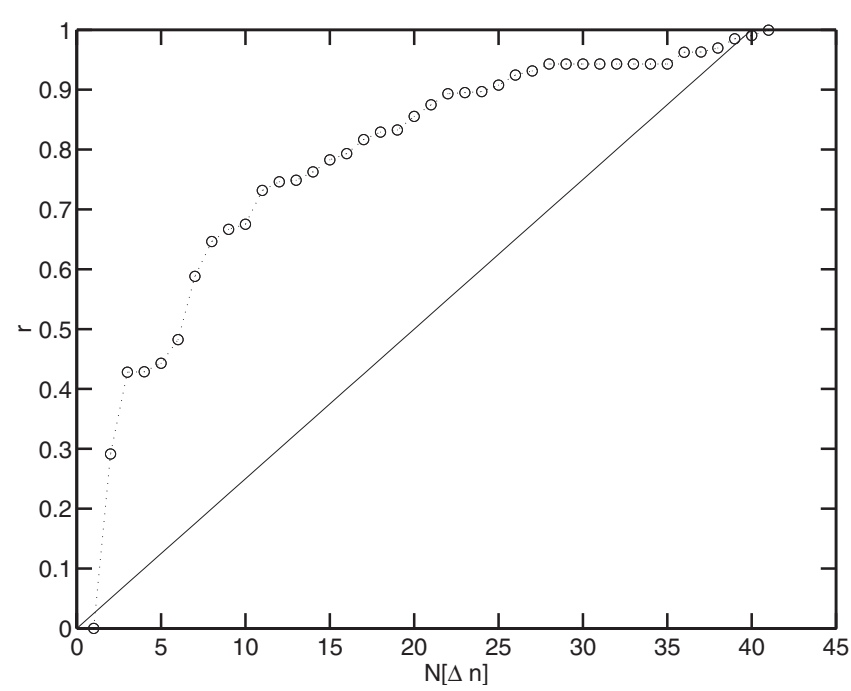

FIG. 9. Plot of signal residue $r$ vs. the range of $n$. The solid line is a plot of $r_{s}=N[\Delta n] / n_{s}$.

By bracketing the residue it is possible to extract information on the error of the mode number and the noise. Thus, for the first three residue ranges we find

$$
\begin{aligned}
& 0 \leq r<r_{s}\left[n+(\Delta n)_{1}\right] \Rightarrow \Delta n=0, \quad r_{n}=r, \\
& r_{s}\left[(\Delta n)_{1}\right] \leq r<r_{s}\left[n+(\Delta n)_{2}\right] \\
& \Rightarrow\left\{\begin{array}{l}
\Delta n=0, r_{n}=r \\
\Delta n=(\Delta n)_{1}, \quad r_{n}=r-r_{s}\left[n+(\Delta n)_{1}\right]
\end{array},\right. \\
& r_{s}\left[n+(\Delta n)_{2}\right] \leq r<r_{s}\left[n+(\Delta n)_{3}\right] \\
& \Rightarrow\left\{\begin{array}{ll}
\Delta n=0, & r_{n}=r \\
\Delta n=(\Delta n)_{1}, & r_{n}=r-r_{s}\left[n+(\Delta n)_{1}\right] \\
\Delta n=(\Delta n)_{2}, & r_{n}=r-r_{s}\left[n+(\Delta n)_{1}\right]
\end{array} .\right.
\end{aligned}
$$

Two observations can be made. First, if $r<r_{s}\left[n+(\Delta n)_{1}\right]$ the correct mode is always identified, and $r_{n}=r$. Second, the range of $n$, denoted here by the operator $N$ acting on the set $\Delta n=\left(1, \ldots, 2 n_{c}\right)$ (i.e., $\left.N[\Delta n]\right)$ increases with increasing residue. In the example above $N[\Delta n]=1,2$, and 3 for the first three lines, respectively, for which $r_{s} \approx 0,0.3$, and 0.4 . More generally, the signal residue and range of $n$ exhibit the property

$$
r_{s} \geq N[\Delta n] / n_{s},
$$

and so the noise residue $r_{n}=r-r_{s}$ obeys

$$
r_{n} \leq r-N[\Delta n] / n_{s} .
$$

Figure 9 is a plot of the residue as a function of $\Delta n$ for the best fit coil combination $\phi=\{0,9,60\}$. The inequality $r_{s} \geq N[\Delta n] / n_{s}$ is clearly visible. This result provides a strategy for the interpretation of alternative Fourier-SVD fits from OMAHA coil data. If only a single mode is present, and $r<0.29$, the correct mode is always identified. If $0.29<r$ $<0.43$, alternative fits is $n \pm \Delta n$.

\section{MAST DATA}

As discussed in the introduction, extensive results from the MAST OMAHA array have now been published. We 


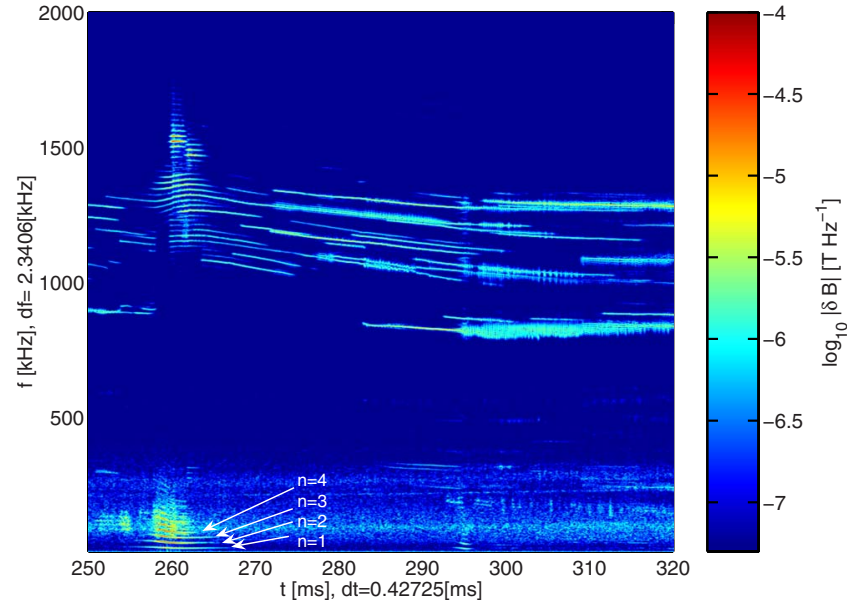

FIG. 10. (Color online) Spectrum of \#17944, showing a multitude of discrete modes, mostly in the frequency range $800 \mathrm{kHz}-1.8 \mathrm{MHz}$.

highlight two such results, ${ }^{3,6}$ which are believed to be observations of modulation between high frequency $(\approx 1 \mathrm{MHz})$ compressional AEs and low frequency $(\approx 20 \mathrm{kHz})$ tearing mode activity. The examples are chosen as they provide good examples of mode activity across a wide range of frequency and amplitude.

Figure 10 is a spectrogram of an OMAHA $B_{\phi}$ coil in MAST discharge \#17944. This discharge was a deuterium plasma with 1.7 MW, $64 \mathrm{keV}$ deuterium neutral beam injected (NBI) applied throughout the discharge lifetime. Throughout the discharge, and below $250 \mathrm{kHz}$, weak signature of plasma noise, possibly $1 / f$ noise, ${ }^{13}$ is visible. In the frequency band $0-200 \mathrm{kHz}$ and $0.8-1.8 \mathrm{MHz}$ coherent individual modes of signal strength down to $|\delta B| \approx 10^{-6} \mathrm{~T}$ can be seen, with lifetimes up to $60 \mathrm{~ms}$. Modes in the high frequency band are believed to be compressional AEs (CAEs). ${ }^{3}$ At around $260 \mathrm{~ms}$, low frequency (20 kHz and harmonics) coherent modes activity of signal strength $|\delta B| \approx 10^{-4.7} \mathrm{~T}$ is observed, with toroidal mode numbers in the range $1 \leq n$ $\leq 4$. Simultaneously, fine structure splitting appears about the $1.3 \mathrm{MHz}$ CAE.

A model for the modulation between frequency components, inspired by similar data from earlier discharge \#9429, has been developed Hole and Appel. ${ }^{6}$ As some of the system analysis presented in Sec. II sheds light on the physics discussion of Hole and Appel, ${ }^{6}$ we revisit these data here. Figure 11 is a spectrogram of an OMAHA $B_{\phi}$ coil in MAST deuterium discharge \#9429. This discharge was a deuterium plasma with 1.25 MW, $45 \mathrm{keV}$ deuterium NBI applied during the current ramp from 100 to $350 \mathrm{~ms}$, and $600 \mathrm{~kW} \mathrm{ECRH}$ applied from 210 to $290 \mathrm{~ms}$. The plasma current plateaus at $780 \mathrm{kA}$, and is in H-mode from $158 \mathrm{~ms}$. Up to $200 \mathrm{~ms}$ there is intermittent bursting high frequency activity. Two upward chirping bands separated by $150 \mathrm{kHz}$ can be identified. Each band consists of a dominant mode ( $n=8$ and $n=9$ for upper and lower band, respectively), and sideband modes of smaller amplitude with $16 \mathrm{kHz}$ spacing. A separate mode analysis ${ }^{13}$ reveals the mode number spacing of these weaker sidebands is $\Delta n=1$. A detailed physics analysis ${ }^{3}$ suggests that these are CAEs, aliased in frequency from 1.4 to $1.9 \mathrm{MHz}$.

In Hole and Appel, ${ }^{6}$ a modulation model was presented

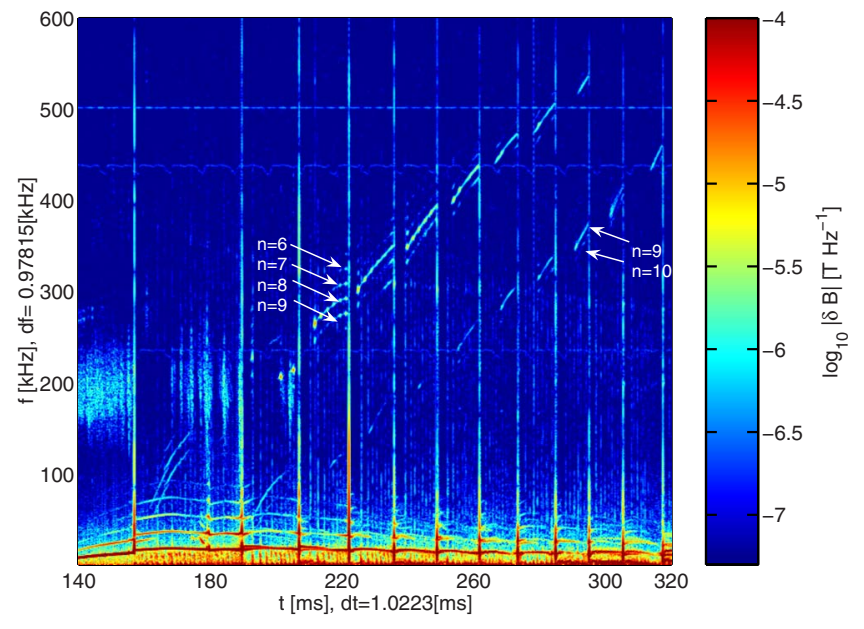

FIG. 11. (Color online) Spectrum of \#9429, showing evidence of both CAE and tearing mode activity. The frequency splitting at low and high frequency match.

to describe the phase relations between the frequency components. A bicoherence analysis revealed that the frequency splitting of CAE modes was consistent with modulation of low frequency modes. Strong evidence was found for frequency and amplitude coupling, with weaker evidence for phase coupling. Phase coupling was consistent only in the presence of an assumed strong phase nonlinearity across the CAE band. In Sec. II we have shown there is indeed evidence for a phase nonlinearity across the 1.4-1.9 MHz band, and that the nonlinearity of the phase response is affected by shielding, at least for $\delta B_{R}$ coils. The nonlinearity in phase also has the same frequency ramp trend: both phases decrease with increasing frequency. The magnitude of the ramp rate is however vastly different. Over the $60 \mathrm{kHz}$ range from 1.68 to $1.74 \mathrm{MHz}$, the inferred phase change of Hole and Appel $^{6}$ is $-4 \pi$, whereas the phase change due to shield effects is $-0.01 \pi$. It hence seems unlikely that the shielding effect discussed here could be responsible for phase nonlinearity of Hole and Appel. ${ }^{6}$

\section{CONCLUSIONS}

We have presented a case study of the design of the new OMAHA in MAST. The new array was motivated by the physics need to resolve AEs and other high frequency modes in MAST. In STs these can be particularly important due to the low field and relatively large Alfvén gap spacing.

Each Mirnov probe was modeled using an equivalent circuit comprising coil, matching elements, and transmission line. Design requirements on the self-resonant frequency were met using an expression for the coil stray capacitance $C_{p}$ and adjusting the wire thickness and insulation. Maximization of the signal transfer function $H_{V, \delta B}$ was obtained by substituting an expression for the coil inductance $L_{p}$, and finding stationary points with respect to the number of coil turns. Finally, characterization of the transmission line, together with a choice of impedance matching for the cable to coil connection determined $L_{p}$, and hence the coil length. One outcome of the coil design analysis is that due to the finite impedance of the transmission line, the magnitude of 
the transfer function saturates well below the self-resonant frequency of the coil. Practically, this saturation frequency can be increased by choosing a transmission line with large impedance. We have also computed the effects of a thin graphite shield on the transfer function, by modeling the shield as the secondary winding of a one-turn solenoidal transformer. The effect of the thin shield on $\delta B_{R}$ is to cause $\left|H_{V, \delta B}\right|$ to roll off at $\approx 250 \mathrm{kHz}$, and the phase of $\left|H_{V, \delta B}\right|$ to ramp down to $-\pi / 2$ at $10 \mathrm{MHz}$.

By inverting a Fourier-SVD mode analysis technique, ${ }^{13}$ we have also developed an algorithm to locate optimal coil toroidal arrangements to resolve modes up to $n=n_{c}$. The analysis technique, which involves numerically searching for coil arrangements that maximize the residue to the fit of all incorrect modes, returns an optimal coil arrangement. We have demonstrated that the optimal coil arrangement of $N$ coils does not change with the addition of more coils, and is therefore a robust placement strategy. By bracketing the residue, we have also been able to construct an algorithm to determine if alternate solutions exist, and if so, the candidate mode numbers.

In summary, we have brought new techniques in stray capacitance modeling, Mirnov coil transmission line theory, design optimization, and coil positioning to an established field. Two important new features of this work are the maximization of the voltage transfer function subject to other design constraints such as impedance matching and the requirement for a coil self-resonant frequency above $5 \mathrm{MHz}$, and an algorithm to choose optimal coil locations for mode identification, obtained by maximizing the residue to the fit of all incorrect mode numbers. This analysis has been used to design and construct OMAHA, a diagnostic now routinely used in MAST. As an example, data from this new array has been used to identify coherent modes structures down to $|\delta B|$ $\approx 10^{-6} \mathrm{~T}$, and across a wide frequency band $(\approx 2 \mathrm{MHz})$.

\section{ACKNOWLEDGMENTS}

This work was partly funded by the Australian National University, the United Kingdom Engineering and Physical
Sciences Research Council, and by the European Communities under the contract of Association between EURATOM and CCFE. The views and opinions expressed herein do not necessarily reflect those of the European Commission.

${ }^{1}$ A. Fasoli, C. Gormenzano, H. L. Berk, B. Breizman, S. Briguglio, D. S. Darrow, N. Gorelenkov, W. W. Heidbrink, A. Jaun, S. V. Konovalov, R. Nazikian, J.-M. Noterdaeme, S. Sharapov, K. Shinohara, D. Testa, K. Tobita, Y. Todo, G. Vlad, and F. Zonca, Nucl. Fusion 47, S264 (2007).

${ }^{2}$ K. L. Wong, Phys. Rev. Lett. 66, 1874 (1991).

${ }^{3}$ The MAST Team, L. C. Appel, T. Fulop, M. J. Hole, H. M. Smith, S. D. Pinches, and R. G. L. Vann, Plasma Phys. Controlled Fusion 50, 115011 (2008).

${ }^{4}$ L. Chen, Plasma Phys. 1, 1519 (1994).

${ }^{5}$ R. Betti and J. P. Freidberg, Phys. Fluids B 4, 1465 (1992).

${ }^{6}$ M. J. Hole and L. C. Appel, Plasma Phys. Controlled Fusion 51, 045022 (2009).

${ }^{7}$ The MAST Team, M. P. Gryaznevich, S. E. Sharapov, M. Lilley, S. D. Pinches, A. R. Field, D. Howell, D. Keeling, R. Martin, H. Meyer, H. Smith, R. Vann, P. Denner, and E. Verwichte, Nucl. Fusion 48, 084003 (2008).

${ }^{8}$ M. Gryaznevich and S. E. Sharapov, Nucl. Fusion 46, S942 (2006).

${ }^{9}$ JET-EFDA Contributors, S. D. Pinches, H. L. Berk, M. P. Gryaznevich, and S. E. Sharapov, Plasma Phys. Controlled Fusion 46, S47 (2004).

${ }^{10}$ K. G. McClements, L. C. Appel, M. J. Hole, and A. Thyagaraja, Nucl. Fusion 42, 1155 (2002).

${ }^{11}$ M. J. Hole and L. C. Appel, IEE Proc.: Circuits Devices Syst. 152, 565 (2005).

${ }^{12}$ L. C. Appel and M. J. Hole, Rev. Sci. Instrum. 76, 093505 (2005).

${ }^{13}$ M. J. Hole and L. C. Appel, Plasma Phys. Controlled Fusion 49, 1971 (2007)

${ }^{14}$ H. A. Wheeler, Proc. IRE 16, 1398 (1928).

${ }^{15}$ N. W. McLachlan, Bessel Functions for Engineers, 2nd ed. (Clarendon, Oxford, 1961).

${ }^{16}$ W. R. Smythe, Static and Dynamic Electricity (McGraw-Hill, New York, 1950), p. 48.

${ }^{17} \mathrm{G}$. J. Greene, "ICRF antenna coupling and wave propagation in a tokamak plasma," Ph.D. thesis, California Institute of Technology, 1984.

${ }^{18}$ Rhodes and Schwarz website, http://www.rsd.de (2005).

${ }^{19}$ E. J. Strait, Rev. Sci. Instrum. 67, 2538 (1996).

${ }^{20} \mathrm{~B}$. Ph. van Milligen and J. A. Jimenez, Proceedings of the 21st EPS Conference on Controlled Fusion and Plasma Physics, Montpellier, France, 1994 (unpublished), Vol. 18B(III), p. 1356. 